\title{
Dynamical calculation of direct muon-transfer rates from thermalized muonic hydrogen to $\mathrm{C}^{6+}$ and $\mathrm{O}^{8+}$
}

\author{
Renat A. Sultanov ${ }^{1,2}$ and Sadhan K. Adhikari ${ }^{1}$ \\ ${ }^{1}$ Instituto de Física Teórica, Universidade Estadual Paulista, 01405-900 São Paulo, São Paulo, Brazil \\ ${ }^{2}$ Department of Physics, Texas A \& M University, College Station, Texas 77843*
}

(Received 29 November 1999; published 18 July 2000)

\begin{abstract}
We perform dynamical calculations of direct muon-transfer rates from thermalized muonic hydrogen isotopes to bare nuclei $\mathrm{C}^{6+}$ and $\mathrm{O}^{8+}$. For these three-body charge-transfer reactions with Coulomb interaction in the final state we use two-component integrodifferential Faddeev-Hahn-type equations in configuration space using close-coupling approximation. To take into account the high polarizability of the muonic hydrogen due to the large charge of the incident nuclei, a polarization potential is included in the elastic channel. The large final-state Coulomb interaction is explicitly included in the transfer channel. The transfer rates so calculated are in good agreement with recent experiments.
\end{abstract}

PACS number(s): 36.10.Dr

\section{INTRODUCTION}

Mainly motivated by the possibility of muon-catalyzed fusion of the hydrogen isotopes, theoretical and experimental investigations of exotic atomic and molecular systems involving negative muon $\left(\mu^{-}\right)$and reactions in such systems continue to be active fields of current research [1]. Particular attention is devoted to the study of the muon-transfer reaction from muonic hydrogen to other elements $X^{Z+}$ with large positive charge $Z$, because such reactions may have large cross sections and rates. If the hydrogen is contaminated by even a small amount of these heavier elements, this may strongly influence the process of muon-catalyzed fusion by hydrogen isotopes. Consequently, there has been considerable recent experimental interest in the study of the muontransfer reaction in the collision of the muonic hydrogen by heavier nuclei, e.g., carbon $\left(\mathrm{C}^{6+}\right)$, oxygen $\left(\mathrm{O}^{8+}\right)$, neon $\left(\mathrm{Ne}^{10+}\right)$, argon $\left(\mathrm{Ar}^{18+}\right)$ [2-6], etc. On the theoretical side, these three-body charge transfer reactions involving a heavy transferred particle such as muon and a strong Coulomb interaction in the final state involving nuclei, such as carbon and oxygen, continue to be challanging problems calling for new investigations.

Experimental study of muon-transfer rates from muonic hydrogen $\mathrm{H}_{\mu}$ to heavier nuclei, such as $\mathrm{Ar}^{18+}, \mathrm{Kr}^{36+}$, and $\mathrm{Xe}^{54+}$, have revealed a smooth $Z$ dependence [7]. In these cases the transfer rate $\lambda_{Z}$ increases linearly with $Z$. Theoretical analyses are also in agreement with this conclusion [8]. However, it has been found in recent experiment that the predicted monotonic $Z$ dependence of the muon-transfer rate is not valid for all $Z[5,9]$. For these transfer rates, pronounced fluctuations have been observed for elements up to $\operatorname{argon}$ contrary to the smooth $Z$ dependence. The experimental muon transfer rates for reactions such as

$$
\left(\mathrm{H}_{\mu}\right)_{1 s}+X^{Z+} \rightarrow\left(X_{\mu}\right)^{(Z-1)+}+\mathrm{H}^{+}
$$

depend in a complicated manner on the charge $Z$ [9]. Here $\mathrm{H}$

\footnotetext{
*Present address.
}

stands for the hydrogen isotope $p$ (proton) or $d$ (deuteron) and $X^{Z+}$ stands for the target nuclei.

Another phenomenon which has not yet found a satisfactory theoretical explanation is the measured isotope effect, e.g., the trend of the direct muon-transfer rate of reaction (1) from $p \mu$ and $d \mu$ to $\mathrm{X}^{Z+}$, where $\mathrm{X}^{Z+}$ represents $\mathrm{C}^{6+}, \mathrm{O}^{8+}$ [3], $\mathrm{Ne}^{10+}$ [9], $\mathrm{Ar}^{18+}$ [2], and $\mathrm{Xe}^{54+}$ [10]. In cases of $\mathrm{O}^{8+}$, $\mathrm{Ar}^{18+}$, and $\mathrm{Xe}^{54+}$ the direct-transfer rate decreases with increasing mass of the hydrogen isotope. Theoretical analyses $[8,11]$ also support this trend. The experimental results for $\mathrm{Ne}^{10}[5,9]$ and sulphur dioxide [3] differ considerably from the theoretical predictions.

In view of this, here we perform quantum dynamical calculation of muon-transfer rates from $p \mu$ to $\mathrm{C}^{6+}$ and $\mathrm{O}^{8+}$ and from $d \mu$ to $\mathrm{O}^{8+}$. For this purpose, we use close-coupling approximation to two-component Faddeev-Hahn-type dynamical equations $[12,13]$. We are currently investigating muon-transfer rates from muonic hydrogen to other heavier elements for a future publication.

It is difficult to perform a quantum dynamical calculation of a charge-transfer reaction. In addition, a theoretical study of the problems above is extremely complicated due to the large charge of these heavy nuclei and the presence of a large number of open channels even at zero incident energy. The large charge of the nuclei leads to a strong polarization of the muonic hydrogen in the initial state and a strong final-state Coulomb repulsion. It is difficult to incorporate these two effects properly in a dynamical calculation. This is why there are no dynamical calculations of these muon-transfer rates.

Although there is a very large number of open channels in this problem, for a given nuclei the muon is transferred predominantly to a few (muonic) atomic labels of the heavy nuclei $[8,11]$. For example, muon is captured mostly in the $n=4$ states of $\mathrm{C}^{6+}$, and $n=5$ states of $\mathrm{O}^{8+}$. Also these transfers take place mostly to the final muonic-atomic states with low angular momenta and transfer rates are negligible for atomic states with angular momenta $l>2$. The correct dynamical formulation should include all the important transfer channels and we included them in a previous study on muon transfer with light nuclear targets [14-17]. How- 
ever, it is quite impossible now to treat even this reduced number of open transfer channels in a dynamical calculation with heavier targets due to convergence difficulties in the presence of the large polarization potential and large finalstate Coulomb interaction mentioned above. Hence in the present treatment we use a two-channel model to calculate transfer to a single final state, where we include the elastic and one transfer channels. Different sets of equations are used for the different final states. Eventually, the total transfer rate is calculated by summing the different contributions.

The two-component Faddeev-Hahn-type equation, we use, allows us to introduce explicitly a polarization potential in the initial channel and the repulsive Coulomb potential in the final channel. This has the advantage of building in the correct asymptotic behavior of the wave function in a loworder close-coupling-type approximation [18]. Hence as in Ref. [14-16] we make a two-state close-coupling approximation to the Faddeev-Hahn-type equation in the present study and find that a numerical solution using the present scheme leads to very encouraging agreement with experimental transfer rates.

In Sec. II we present our formalism. Numerical results for muon-transfer rates from muonic hydrogen to carbon and oxygen are given in Sec. III and compared with those of other investigations. In Sec. IV we present a summary and outlook.

\section{THEORETICAL FORMULATION}

The theoretical framework for the present study will be based on the formalism developed in Ref. [15] which was used for the study of muon transfer from muonic hydrogen atoms to light charged nuclei, such as $\mathrm{He}^{2+}$ and $\mathrm{Li}^{3+}$. Here we shall perform a similar study with heavier charged nuclei, such as $\mathrm{C}^{6+}$ and $\mathrm{O}^{8+}$. The presence of the strong Coulomb interaction and the associated large polarization interaction make the present calculational scheme far more complicated theoretically and numerically compared to that of Ref. [15]. The formalism of Ref. [15] is a generalization over that of Ref. [14] for charge-transfer reaction with no final-state Coulomb interaction. In the dynamical equations in Ref. [15] the final-state Coulomb interaction is explicitly included in the transfer channel. In addition, in the present work we explicitly include a polarization potential in the elastic channel. In a coupled-channel approach for atomic processes, the coupling to infinite number of $p$-wave states is responsible for generating the polarization potential [19]. As it is impossible to include all such states in a numerical scheme, the commonly accepted procedure is to replace these coupling terms by a polarization potential as in Ref. [20]. This idea has been recently used successfully in antiproton-hydrogen and antihydrogen-hydrogen reactions [21]. Next we describe the dynamical equations we use based on the close-coupling approximation to Faddeev-Hahn-type two-component equations [14].

We use units $e=\hbar=m_{\mu}=1$, where $m_{\mu}(e)$ is the muonic mass (charge), and denote, the heavy nuclei $\left(\mathrm{C}^{6+}\right.$ or $\left.\mathrm{O}^{8+}\right)$ by 1 , the hydrogen isotopes by 2 and muon by 3 . Below the three-body breakup threshold, following two-cluster asymptotic configurations are possible in the system 123: (23) -1 and (13) -2 . These two configurations correspond to two distinct physical channels, denoted by 1 and 2 , respectively. These configurations are determined by the Jacobi coordinates $\quad\left(\vec{r}_{j 3}, \vec{\rho}_{k}\right): \quad \vec{r}_{13}=\vec{r}_{3}-\vec{r}_{1}, \vec{\rho}_{2}=\left(\vec{r}_{3}+m_{1} \vec{r}_{1}\right) /(1$ $\left.+m_{1}\right)-\vec{r}_{2}, \quad \vec{r}_{23}=\vec{r}_{3}-\vec{r}_{2}, \quad \vec{\rho}_{1}=\left(\vec{r}_{3}+m_{2} \vec{r}_{2}\right) /\left(1+m_{2}\right)-\vec{r}_{1}$, where $\vec{r}_{i}, m_{i}(i=1,2,3$,$) are coordinates and masses of the$ particles, respectively.

Let us introduce the total three-body wave function as a sum of two components

$$
\Psi\left(\vec{r}_{1}, \vec{r}_{2}, \vec{r}_{3}\right)=\Psi_{1}\left(\vec{r}_{23}, \vec{\rho}_{1}\right)+\Psi_{2}\left(\vec{r}_{13}, \vec{\rho}_{2}\right),
$$

where $\Psi_{1}\left(\vec{r}_{23}, \vec{\rho}_{1}\right)$ is quadratically integrable over the variable $\vec{r}_{23}$, and $\Psi_{2}\left(\vec{r}_{13}, \vec{\rho}_{2}\right)$ over the variable $\vec{r}_{13}$. The components $\Psi_{1}$ and $\Psi_{2}$ carry the asymptotic boundary condition for channels 1 and 2, respectively. The second component is responsible for pure Coulomb interaction in the final state. These components satisfy the following set of two coupled equations

$$
\begin{aligned}
& {\left[E-H_{0}-V_{23}\left(\vec{r}_{23}\right)-U_{\mathrm{pol}}\left(\vec{\rho}_{1}\right)\right] \Psi_{1}\left(\vec{r}_{23}, \vec{\rho}_{1}\right)} \\
& \quad=\left[V_{23}\left(\vec{r}_{23}\right)+V_{12}\left(\vec{r}_{12}\right)-U_{\mathrm{C}}\left(\vec{\rho}_{2}\right)\right] \Psi_{2}\left(\vec{r}_{13}, \vec{\rho}_{2}\right), \\
& {\left[E-H_{0}-V_{13}\left(\vec{r}_{13}\right)-U_{\mathrm{C}}\left(\vec{\rho}_{2}\right)\right] \Psi_{2}\left(\vec{r}_{13}, \vec{\rho}_{2}\right)} \\
& \quad=\left[V_{13}\left(\vec{r}_{13}\right)+V_{12}\left(\vec{r}_{12}\right)-U_{\mathrm{pol}}\left(\vec{\rho}_{1}\right)\right] \Psi_{1}\left(\vec{r}_{23}, \vec{\rho}_{1}\right),
\end{aligned}
$$

where $E$ is the center-of-mass energy, $H_{0}$ is the total kinetic energy operator, $V_{i j}\left(\vec{r}_{i j}\right)$ pair potentials $(i \neq j=1,2,3), U_{\mathrm{C}}$ is the final-state Coulomb interaction given by

$$
U_{\mathrm{C}}\left(\vec{\rho}_{2}\right)=\frac{(Z-1) Z^{\prime}}{\rho_{2}},
$$

with $Z$ the charge of the heavy nuclei and $Z^{\prime}(=1)$ the charge of the hydrogen isotope. Here $U_{\mathrm{pol}}$ is the polarization potential given by [11]

$$
U_{\mathrm{pol}}\left(\vec{\rho}_{1}\right)=-\frac{9 Z^{2}}{4 \rho_{1}^{4}}, \quad \rho_{1}>\Lambda
$$

and zero otherwise. The value of the cutoff parameter $\Lambda$ has to be chosen appropriately (see Sec. III). By adding Eqs. (3) and (4) we find that they are equivalent to the Schrödinger equation.

Distortion potential has been very useful in model and phenomenological description of reaction and scattering in nuclear [22] and atomic physics [23]. Although such distortion potentials are unnecessary in a complete solution of the Schrödinger equation, they enhance the agreement with experiment in a simplified model description. For example, a long-range polarization (distortion) potential has been routinely used in electron-atom scattering $[20,24]$. Such a distortion potential arising from the polarization of the muonic hydrogen isotope due to the bare nuclei is effective in the initial channel and has been included in Eq. (3). This polar- 
ization potential for $\mathrm{C}^{6+}$ or $\mathrm{O}^{8+}$ is much stronger and its effect on cross sections much more pronounced than in the case of electron scattering. Hence, for obtaining a better agreement with experiment in a model calculation it is prudent to include the polarization potential in the elastic channel. There should also be such a polarization potential in the final channel. However, by far the most important interaction in the final channel is the Coulomb potential between the proton (or deuteron) and the charged muonic atom $\left(X_{\mu}\right)^{(Z-1)+}$. This Coulomb (distortion) potential has also been explicitly included in Eq. (4). This will help in obtaining a realistic description of the transfer process as we shall find in the following.

Because of the strong final-state Coulomb interaction in the present muon-transfer problems it is very difficult to develop and solve successfully multichannel models based on Eqs. (3) and (4) above as in Ref. [15]. Hence, for solving Eqs. (3) and (4) we expand the wave function components in terms of bound states in initial and final channels, and project these equations on these bound states. The expansion of the wave function is given by

$$
\begin{gathered}
\Psi_{1}\left(\vec{r}_{23}, \vec{\rho}_{1}\right) \approx \frac{f_{1 s}^{(1)}\left(\rho_{1}\right)}{\rho_{1}} R_{1 s, \mu_{1}}^{\left(Z^{\prime}\right)}\left(\left|\vec{r}_{23}\right|\right) / 4 \pi, \\
\Psi_{2}\left(\vec{r}_{13}, \vec{\rho}_{2}\right) \approx \frac{f_{n l \mathcal{L}}^{(2)}\left(\rho_{2}\right)}{\rho_{2}} R_{n l, \mu_{2}}^{(Z)}\left(\left|\vec{r}_{13}\right|\right)\left\{Y_{\mathcal{L}}\left(\hat{\rho}_{2}\right) \otimes Y_{l}\left(\hat{r}_{13}\right)\right\}_{00},
\end{gathered}
$$

where $n l \mathcal{L}$ are quantum numbers of the three-body final state, $\mu_{1}=m_{3} m_{2} /\left(m_{3}+m_{2}\right), \mu_{2}=m_{3} m_{1} /\left(m_{3}+m_{1}\right), Y_{l m}$ 's are the spherical harmonics, $R_{n l, \mu_{i}}^{(Z)}(|\vec{r}|)$ is the radial part of the hydrogenlike bound-state wave function for reduced mass $\mu_{i}$ and charge $Z, f_{1 s}^{(1)}\left(\rho_{1}\right)$ and $f_{n l \mathcal{L}}^{(2)}\left(\rho_{2}\right)$ are the unknown expansion coefficients. This prescription is similar to that adopted in the close-coupling approximation. After a proper angular momentum projection, the set of two-coupled integrodifferential equations for the unknown expansion functions can be written as

$$
\begin{aligned}
& {\left[\left(k_{1}^{(1)}\right)^{2}+\frac{\partial^{2}}{\partial \rho_{1}^{2}}-2 M_{1} U_{\mathrm{pol}}\left(\vec{\rho}_{1}\right)\right] f_{1 s}^{(1)}\left(\rho_{1}\right)} \\
& =g_{1} \sqrt{(2 \mathcal{L}+1)} \int_{0}^{\infty} d \rho_{2} f_{n l \mathcal{L}}^{(2)}\left(\rho_{2}\right) \int_{0}^{\pi} d \omega \sin \omega \\
& \quad \times R_{1 s, \mu_{1}}^{\left(Z^{\prime}\right)}\left(\left|\vec{r}_{23}\right|\right)\left(-\frac{Z^{\prime}}{\left|\vec{r}_{23}\right|}+\frac{Z}{\left|\vec{r}_{12}\right|}-U_{\mathrm{C}}\left(\vec{\rho}_{2}\right)\right) \\
& \quad \times R_{n l, \mu_{2}}^{(Z)}\left(\left|\vec{r}_{13}\right|\right) \rho_{1} \rho_{2} C_{\mathcal{L} 0 l 0}^{00} Y_{l m}\left(\nu_{2}, \pi\right) / \sqrt{4 \pi},
\end{aligned}
$$

$$
\begin{gathered}
{\left[\left(k_{n}^{(2)}\right)^{2}+\frac{\partial^{2}}{\partial \rho_{2}^{2}}-\frac{\mathcal{L}(\mathcal{L}+1)}{\rho_{2}^{2}}-2 M_{2} U_{\mathrm{C}}\left(\vec{\rho}_{2}\right)\right] f_{n l \mathcal{L}}^{(2)}\left(\rho_{2}\right)} \\
=g_{2} \sqrt{(2 \mathcal{L}+1)} \int_{0}^{\infty} d \rho_{1} f_{1 s}^{(1)}\left(\rho_{1}\right) \int_{0}^{\pi} d \omega \sin \omega \\
\quad \times R_{n l, \mu_{2}}^{(Z)}\left(\left|\vec{r}_{13}\right|\right)\left(-\frac{Z}{\left|\vec{r}_{13}\right|}+\frac{Z}{\left|\vec{r}_{12}\right|}-U_{\mathrm{pol}}\left(\vec{\rho}_{1}\right)\right) \\
\quad \times R_{1 s, \mu_{1}}^{\left(Z^{\prime}\right)}\left(\left|\vec{r}_{23}\right|\right) \rho_{2} \rho_{1} C_{\mathcal{L} 0 l 0}^{00} Y_{l m}\left(\nu_{1}, \pi\right) / \sqrt{4 \pi}
\end{gathered}
$$

Here $k_{1}^{(1)}=\sqrt{2 M_{1}\left(E-E_{1 s}^{(2)}\right)}, \quad k_{n}^{(2)}=\sqrt{2 M_{2}\left(E-E_{n}^{(1)}\right)}$ with $M_{1}^{-1}=m_{1}^{-1}+\left(1+m_{2}\right)^{-1}$ and $M_{2}^{-1}=m_{2}^{-1}+\left(1+m_{1}\right)^{-1}, E_{n}^{(j)}$ is the binding energy of pair $(j 3)$ and $g_{j}=4 \pi M_{j} / \gamma^{3}(j$ $=1,2), \quad \gamma=1-m_{1} m_{2} /\left[\left(1+m_{1}\right)\left(1+m_{2}\right)\right], \quad C_{\mathcal{L} 0 l m}^{L m} \quad$ the Clebsch-Gordon coefficient, $\omega$ is the angle between the Jacobi coordinates $\vec{\rho}_{1}$ and $\vec{\rho}_{2}, \nu_{1}$ is the angle between $\vec{r}_{23}$ and $\vec{\rho}_{1}, \nu_{2}$ is the angle between $\vec{r}_{13}$ and $\vec{\rho}_{2}$.

We find that after the projection of the Faddeev-Hahntype equations (3) and (4) on the basis states, the initial-state polarization and the final-state Coulomb potentials survive on the left-hand-side of the resultant equations (9) and (10). The presence of the explicit Coulomb potential in the final channel will automatically yield the correct physical Coulomb-wave boundary condition in that channel. The explicit inclusion of the polarization potential, although has no effect on the boundary condition in the initial channel, substantially improves the results of the truncated model calculation based on Eqs. (9) and (10) as we shall see in the next section.

To find unique solution to Eqs. (9)-(10), appropriate boundary conditions are to be considered. We impose the usual condition of regularity at the origin $f_{1 s}^{(1)}(0)=0$ and $f_{n l \mathcal{L}}^{(2)}(0)=0$. Also for the present scattering problem with 1 $+(23)$ as the initial state, in the asymptotic region, two solutions to Eqs. (9)-(10) satisfy the following boundary conditions:

$$
\begin{aligned}
f_{1 s}^{(1)}\left(\rho_{1}\right) \underset{\rho_{1} \rightarrow+\infty}{\sim} \sin \left(k_{1}^{(1)} \rho_{1}\right)+K_{11}^{n l} \cos \left(k_{1}^{(1)} \rho_{1}\right), \\
f_{n l \mathcal{L}}^{(2)}\left(\rho_{2}\right) \underset{\rho_{2} \rightarrow+\infty}{\sim} \sqrt{v_{1} / v_{2}} K_{12}^{n l} \\
\quad \times \cos \left(k_{1}^{(2)} \rho_{2}-\eta / 2 k_{1}^{(2)} \ln 2 k_{1}^{(2)} \rho_{2}-\pi \mathcal{L} / 2\right),
\end{aligned}
$$

where $K_{i j}^{n l}$ are the appropriate coefficients. For scattering with $2+(13)$ as the initial state, we have the following conditions:

$$
\begin{gathered}
f_{1 s}^{(1)}\left(\rho_{1}\right) \underset{\rho_{1} \rightarrow+\infty}{\sim} \sqrt{v_{2} / v_{1}} K_{21}^{n l} \cos \left(k_{1}^{(1)} \rho_{1}\right), \\
f_{n l \mathcal{L}}^{(2)}\left(\rho_{2}\right) \underset{\rho_{2} \rightarrow+\infty}{\sim} \sin \left(k_{1}^{(2)} \rho_{2}-\eta / 2 k_{1}^{(2)} \ln 2 k_{1}^{(2)} \rho_{2}-\pi \mathcal{L} / 2\right) \\
+K_{22}^{n l} \cos \left(k_{1}^{(2)} \rho_{2}-\eta / 2 k_{1}^{(2)} \ln 2 k_{1}^{(2)} \rho_{2}-\pi \mathcal{L} / 2\right),
\end{gathered}
$$


where $v_{i}(i=1,2)$ is velocity in channel $i$. The Coulomb parameter in the second transfer channel is $\eta=2 M_{2}(Z$ $-1) / k_{n}^{(2)}$ [23]. The coefficients $K_{i j}^{n l}$ are obtained from the numerical solution of the Faddeev-Hahn-type equations. The cross sections are given by

$$
\sigma_{1 s \rightarrow n l}^{\mathrm{tr}}=\frac{4 \pi}{k^{(1) 2}} \frac{\left(K_{12}^{n l}\right)^{2}}{(D-1)^{2}+\left(K_{11}^{n l}+K_{22}^{n l}\right)^{2}},
$$

where $\quad D=K_{11}^{n l} K_{22}^{n l}-K_{12}^{n l} K_{21}^{n l}$. When $\quad k^{(1)} \rightarrow 0: \quad \sigma_{1 s \rightarrow n l}^{\mathrm{tr}}$ $\sim 1 / k_{1}^{(1)}$. The transfer rates are defined by

$$
\lambda_{1 s \rightarrow n l}^{\operatorname{tr}}=\sigma_{1 s \rightarrow n l}^{\operatorname{tr}} v_{1} N_{0},
$$

with $v_{1}$ being the relative velocity of the incident fragments and $N_{0}$ the liquid-hydrogen density chosen here as 4.25 $\times 10^{22} \mathrm{~cm}^{-3}$, because $\lambda^{\operatorname{tr}}\left(k^{(1)} \rightarrow 0\right) \sim$ const. In our model approach the total muon transfer rate is

$$
\lambda_{\mathrm{tot}}^{\mathrm{tr}}=\sum \lambda_{1 s \rightarrow n l}^{\mathrm{tr}}
$$

\section{NUMERICAL RESULTS}

We employ muonic atomic unit: distances are measured in units of $a_{\mu}$, where $a_{\mu}$ is the radius of muonic hydrogen atom. The integrodifferential equations are solved by discretizing them into a linear system of equations. The integrals in Eqs. (9) and (10) are discretized using the trapezoidal rule and the partial derivatives are discretized using a three-point rule [25]. The discretized equation is subsequently solved by Gauss elimination method. As we are concerned with the low-energy limit, only the total angular momentum $L=0$ is taken into account. Even at zero incident energy, the transfer channels are open and their wave functions are rapidly oscillating Coulomb waves. In order to get a converged solution we needed a large number of discretization points. More points are taken near the origin where the interaction potentials are large; a smaller number of points are needed at large distances.

First we solved the system of equations without the polarization potential in the incident channel. However, the final-state Coulomb interaction is correctly represented in our model. In this case it is relatively easy to obtain the numerical convergence for the system of equations which includes the elastic channel and one transfer channel at a time. Finally, the total transfer cross section is calculated by adding the results of different two-channel contributions. Without the polarization potential, we needed up to 700 discretization points adequately distributed between 0 and $50 a_{\mu}$. Near the origin we took up to 60 equally spaced points per unit interval $\left(a_{\mu}\right)$.

It was more difficult to obtain convergence with the polarization potential. The polarization potential (6) is taken to be zero at small distances below the cut off $\Lambda$. In this case to get numerical convergence we had to integrate to very large distances-up to $300 a_{\mu}$. We needed up to 2000 discretization points to obtain convergence. Again we needed more points near the origin and less at large distances. For ex- ample, near the origin we took up to 60 equally spaced points per unit length interval $a_{\mu}$; in the intermediate region $\left(\rho=10-20 a_{\mu}\right)$ we took up to 15 equally spaced points per unit length interval, and in the asymptotic region ( $\rho$ $=20-300 a_{\mu}$ ) we took up to five equally spaced points per unit length interval. It is well-known that the results for the cross section is sensitive to the value of the cutoff $\Lambda$ of the polarization potential. The short-range potential of the present problem extends to about $25 a_{\mu}$. We considered the polarization potential in the asymptotic region $\rho_{1}>\Lambda$ $\simeq 75 a_{\mu}$. For a small variation of $\Lambda$ in this region from $75 a_{\mu}$ to about $120 a_{\mu}$, we find the transfer cross sections to be reasonably constant and the reported transfer cross sections of this study are the averages of these cross sections. If $\Lambda$ is increased past $120 a_{\mu}$, the effect of the polarization potential on the cross sections gradually decreases and finally disappears. If $\Lambda$ is decreased much below $75 a_{\mu}$, the cross sections become rapidly varying function of $\Lambda$ and could become unphysically large. The range of $\Lambda$ values (here between $75 a_{\mu}$ and $120 a_{\mu}$ ) for which the cross sections are slowly varying smooth functions should increase with the charge of the bare nucleus.

We present partial muon-transfer rates $\lambda_{n l}^{\mathrm{tr}}$ and total transfer rates $\lambda_{\text {tot }}^{\text {tr }}$ calculated using the formulation of last section. In this work using the model of Sec. II we calculate the low-energy muon-transfer rates from $(p \mu)_{1 s}$ to $\mathrm{C}^{6+}$ and $\mathrm{O}^{8+}$ and from $(d \mu)_{1 s}$ to $\mathrm{O}^{8+}$. From other theoretical $[8,11]$ investigations it was concluded that in the case of $\mathrm{C}^{6+}$ the transfer takes place predominantly to the $n=4$ state and for $\mathrm{O}^{8+}$ it happens to the $n=5$ state, which is also found to be true in our model calculation. Hence in this work we only present rates for the $l=0,1,2$ states of the $n=4$ and 5 orbitals of carbon and oxygen, respectively. The contribution of the higher angular momentum states to the total transfer cross section is very small. Numerically converged results were obtained in these cases. The low energy partial rates $\lambda_{1 s \rightarrow n l}^{\mathrm{tr}} / 10^{10} \mathrm{sec}^{-1}$ and total rates $\lambda_{\text {tot }}^{\mathrm{tr}} / 10^{10} \mathrm{sec}^{-1}$ are presented in Tables I, II, and III together with the results of other theoretical and experimental works.

First we comment on the results in Table I for muon transfer from $(p \mu)_{1 s}$ to $\mathrm{C}^{6+}$. The partial transfer rates without the polarization potential increases with decreasing center-of-mass energy $E$ and saturates to a constant value for $E<0.04 \mathrm{eV}$ for $4 s, 4 p$, and $4 d$ states of muonic carbon. In the case of $\mathrm{C}^{6+}$ the transfer takes place predominantly to the $4 s$ state. These qualitative behaviors are also true after the inclusion of the polarization potential and were also true in a previous theoretical study. However, after the inclusion of the polarization potential the $4 s$ and $4 p$ transfer rates are enhanced by about a factor of 1.5. The present total transfer rate of $8.5 \times 10^{10} \mathrm{sec}^{-1}$ is about three times larger than the previous theoretical calculation of $2.8 \times 10^{10} \mathrm{sec}^{-1}$ [11]. We quote two experimental results in this case: (9.5 \pm 0.5$)$ $\times 10^{10} \mathrm{sec}^{-1}[2]$ and $(5.1 \pm 1.0) \times 10^{10} \mathrm{sec}^{-1}$ [7]. The present theoretical result lies in between these two somewhat conflicting experimental results.

In the case of muon transfer from $(p \mu)_{1 s}$ to $\mathrm{O}^{8+}$, we find from Table II that transfer takes place predominantly to the 
TABLE I. Low-energy partial $\lambda_{1 s \rightarrow n l}^{\mathrm{tr}} / 10^{10} \mathrm{sec}^{-1}$ and total $\lambda_{\text {tot }}^{\mathrm{tr}} / 10^{10} \mathrm{sec}^{-1}$ muon transfer rates reduced to liquid-hydrogen density $N_{0}=4.25 \times 10^{22} \mathrm{~cm}^{-3}$ from muonic hydrogen $(p \mu)_{1 s}$ to hydrogen-like excited state of muonic carbon $\left(\mathrm{C}_{\mu}\right)_{n=4}^{5+}$.

\begin{tabular}{|c|c|c|c|c|c|c|c|c|}
\hline \multirow{2}{*}{$\begin{array}{l}\text { Energy } \\
E(\mathrm{eV})\end{array}$} & \multirow[b]{2}{*}{$(n l)$} & \multicolumn{2}{|c|}{$U_{\mathrm{pol}}\left(\rho_{1}\right)=0$} & \multicolumn{2}{|c|}{ With polarization } & \multirow{2}{*}{$\begin{array}{c}\text { Theory } \\
{[11]}\end{array}$} & \multicolumn{2}{|c|}{ Experiment } \\
\hline & & $\lambda_{1 s \rightarrow n l}^{\operatorname{tr}}$ & $\lambda_{\text {tot }}^{\operatorname{tr}}$ & $\lambda_{1 s \rightarrow n l}^{\operatorname{tr}}$ & $\lambda_{\text {tot }}^{\operatorname{tr}}$ & & [7] & [2] \\
\hline & $4 s$ & 3.4 & & $5.2 \pm 0.2$ & & & & \\
\hline \multirow[t]{3}{*}{0.04} & $4 p$ & 2.1 & 5.5 & $3.2 \pm 0.5$ & $8.5 \pm 0.7$ & 2.8 & $5.1 \pm 1.0$ & $9.5 \pm 0.5$ \\
\hline & $4 d$ & 0.05 & & 0.1 & & & & \\
\hline & $4 s$ & 2.1 & & $2.8 \pm 0.2$ & & & & \\
\hline \multirow[t]{3}{*}{0.1} & $4 p$ & 1.1 & 3.2 & $1.5 \pm 0.2$ & $4.3 \pm 0.4$ & & & \\
\hline & $4 d$ & $\sim 0$ & & $\sim 0$ & & & & \\
\hline & $4 s$ & 1.2 & & $1.6 \pm 0.1$ & & & & \\
\hline \multirow[t]{2}{*}{0.5} & $4 p$ & 0.4 & 1.6 & $0.7 \pm 0.1$ & $2.3 \pm 0.2$ & & & \\
\hline & $4 d$ & $\sim 0$ & & $\sim 0$ & & & & \\
\hline
\end{tabular}

$5 s$ and $5 p$ states of muonic oxygen. Again the transfer rates saturate and attain constant values for $E<0.04 \mathrm{eV}$. The transfer rate is higher in the $5 s$ state and lowest in the $5 d$ state. This behavior remain true after the inclusion of the polarization potential, when the transfer rate to the $5 p$ state increases by a factor of more than 2 whereas the contribution to the $5 s$ state increases by a factor of 1.5 . The present total transfer rate of $(7.7 \pm 0.5) \times 10^{10} \mathrm{sec}^{-1}$ is about 1.5 times larger than the previous theoretical calculation of 5.6 $\times 10^{10} \mathrm{sec}^{-1}[11]$ and in reasonable agreement with the recent experimental rate of $(8.5 \pm 0.2) \times 10^{10} \mathrm{sec}^{-1}[4]$.

Finally, in the case of muon transfer from $(d \mu)_{1 s}$ to $\mathrm{O}^{8+}$, we find from Table III that transfer also takes place predominantly to the $5 s$ state of muonic oxygen. The contribution to the transfer rate due to $5 s$ state is two times as large as the contribution due to the $5 p$ state. Again the transfer rate saturate and attain a constant value for $E<0.04 \mathrm{eV}$. After the inclusion of the polarization potential the transfer rate to the $5 s$ state increases by a factor of 1.5 . The present total transfer rate of $(4.4 \pm 0.6) \times 10^{10} \mathrm{sec}^{-1}$ is in reasonable agreement with the recent experimental rate of $5.5 \times 10^{10} \mathrm{sec}^{-1}$ [3].

The $Z$ dependence of the transfer rates from a specific hydrogen isotope to $X^{Z+}$ has been a subject of interest. Al- though for large $Z$ these rates increase linearly with $Z$, there is no general behavior for small $Z$. The most recent experimental transfer rates decrease when we move from the system $p \mu-\mathrm{C}^{6+}$ to $p \mu-\mathrm{O}^{8+}[2,4]$. Through our dynamical calculation we have been able to reproduce this behavior. Our calculation is also consistent with the experimentally observed isotope effect, e.g., the transfer rate decreases when we move from $p \mu-\mathrm{O}^{8+}$ to $d \mu-\mathrm{O}^{8+}[3,4]$.

\section{CONCLUSION}

We have studied muon-transfer reactions from muonic hydrogen to carbon and oxygen nuclei employing a full quantum-mechanical few-body description of rearrangement scattering by solving the Faddeev-Hahn-type equations using close-coupling approximation. To provide the correct asymptotic form of the wave function in the transfer channel, the final-state Coulomb interaction has been incorporated directly into the equations. We also included a polarization potential at large distances in the initial channel. It is shown that in the present approach, the application of a closecoupling-type ansatz leads to satisfactory results for direct muon-transfer reactions from muonic hydrogen to $\mathrm{C}^{6+}$ and

TABLE II. Low-energy partial $\lambda_{1 s \rightarrow n l}^{\mathrm{tr}} / 10^{10} \mathrm{sec}^{-1}$ and total $\lambda_{\mathrm{tot}}^{\mathrm{tr}} / 10^{10} \mathrm{sec}^{-1}$ muon transfer rates reduced to liquid-hydrogen density $N_{0}=4.25 \times 10^{22} \mathrm{~cm}^{-3}$ from muonic hydrogen $(p \mu)_{1 s}$ to hydrogenlike excited state of muonic oxygen $\left(\mathrm{O}_{\mu}\right)_{n=5}^{7+}$.

\begin{tabular}{|c|c|c|c|c|c|c|c|c|}
\hline \multirow{2}{*}{$\begin{array}{l}\text { Energy } \\
E(\mathrm{eV})\end{array}$} & \multirow[b]{2}{*}{$(n l)$} & \multicolumn{2}{|c|}{$U_{\mathrm{pol}}\left(\rho_{1}\right)=0$} & \multicolumn{2}{|c|}{ With polarization } & \multirow{2}{*}{$\begin{array}{c}\text { Theory } \\
{[11]}\end{array}$} & \multicolumn{2}{|c|}{ Experiment } \\
\hline & & $\lambda_{1 s \rightarrow n l}^{\operatorname{tr}}$ & $\lambda_{\text {tot }}^{\mathrm{tr}}$ & $\lambda_{1 s \rightarrow n l}^{\operatorname{tr}}$ & $\lambda_{\text {tot }}^{\mathrm{tr}}$ & & [3] & [4] \\
\hline & $5 s$ & 3.5 & & $5.5 \pm 0.2$ & & & & \\
\hline \multirow[t]{3}{*}{0.04} & $5 p$ & 0.8 & 4.35 & $2.1 \pm 0.2$ & $7.7 \pm 0.5$ & 5.6 & 8.3 & $8.5 \pm 0.2$ \\
\hline & $5 d$ & 0.05 & & $0.1 \pm 0.05$ & & & & \\
\hline & $5 s$ & 3.1 & & $5.0 \pm 0.2$ & & & & \\
\hline \multirow[t]{3}{*}{0.1} & $5 p$ & 0.7 & 3.8 & $1.7 \pm 0.2$ & $6.8 \pm 0.5$ & & & \\
\hline & $5 d$ & 0.02 & & 0.05 & & & & \\
\hline & $5 s$ & 2.0 & & $2.9 \pm 0.1$ & & & & \\
\hline \multirow[t]{2}{*}{0.5} & $5 p$ & 0.2 & 2.2 & $1.0 \pm 0.1$ & $3.9 \pm 0.2$ & & & \\
\hline & $5 d$ & $\sim 0$ & & $\sim 0$ & & & & \\
\hline
\end{tabular}


TABLE III. Low-energy partial $\lambda_{1 s \rightarrow n l}^{\text {tr }} / 10^{10} \mathrm{sec}^{-1}$ and total $\lambda_{\text {tot }}^{\mathrm{tr}} / 10^{10} \mathrm{sec}^{-1}$ muon transfer rates reduced to liquid-hydrogen density $N_{0}=4.25 \times 10^{22} \mathrm{~cm}^{-3}$ from muonic hydrogen $(d \mu)_{1 s}$ to hydrogenlike excited state of muonic oxygen $\left(\mathrm{O}_{\mu}\right)_{n=5}^{7+}$.

\begin{tabular}{lcccccc}
\hline \hline $\begin{array}{c}\text { Energy } \\
E(\mathrm{eV})\end{array}$ & \multicolumn{5}{c}{$U_{\mathrm{pol}}\left(\rho_{1}\right)=0$} & \multicolumn{2}{c}{ With polarization } & Experiment \\
$\lambda_{1 s \rightarrow n l}^{\mathrm{tr}}$ & $\lambda_{\text {tot }}^{\mathrm{tr}}$ & $\lambda_{1 s \rightarrow n l}^{\mathrm{tr}}$ & $\lambda_{\text {tot }}^{\mathrm{tr}}$ & {$[3]$} \\
\hline \multirow{4}{*}{0.04} & $5 s$ & 1.9 & & $2.9 \pm 0.3$ & & \\
& $5 p$ & 0.8 & 2.7 & $1.5 \pm 0.3$ & $4.4 \pm 0.6$ & 5.5 \\
& $5 d$ & $\leqslant 0.01$ & & $\sim 0$ & & \\
0.1 & $5 s$ & 1.1 & & $1.8 \pm 0.2$ & & \\
& $5 p$ & 0.5 & 1.6 & $0.7 \pm 0.2$ & $2.5 \pm 0.4$ & \\
& $5 d$ & $\leqslant 0.01$ & & $\sim 0$ & & \\
0.5 & $5 s$ & 0.7 & & $1.0 \pm 0.2$ & & \\
& $5 p$ & 0.1 & 0.8 & $0.2 \pm 0.1$ & $1.2 \pm 0.3$ & \\
& $5 d$ & $\leqslant 0.01$ & & $\sim 0$ & & \\
\hline \hline
\end{tabular}

$\mathrm{O}^{8+}$. In the case of muon transfer from $(p \mu)_{1 s}$ to $\mathrm{C}^{6+}$ the present transfer rate to the $4 s$ state of muonic carbon is about 1.5 times larger than that to the $4 p$ state. For muon transfer from $(p \mu)_{1 s}$ to $\mathrm{O}^{8+}$, the present transfer rate to the $5 s$ state of oxygen is about twice as large as that to the $5 p$ state. The present rates are much larger by factors of about two to three compared to the calculation of Ref. [11]. Finally, in the case of muon transfer from $(d \mu)_{1 s}$ to $\mathrm{O}^{8+}$, the present transfer rate to the $5 s$ state is large compared to that to the $5 p$ state. In all cases the inclusion of the polarization potential improves the agreement with experiment and our final transfer rates are in encouraging agreement with recent experiments $[2-4]$. The present rates for oxygen from $(p \mu)_{1 s}$ and $(d \mu)_{1 s}$ are in agreement with the observed isotope effect [3]: the transfer rate increases with the decrease of the mass of the hydrogen isotope. Because of the present promising results for the muon-transfer rates for $Z=6$ and $Z=8$ it seems useful to make future applications of the present formulation for larger targets. Calculations involving nuclei of higher charges $\left(\mathrm{Ne}^{10+}, \mathrm{S}^{16+}, \mathrm{Ar}^{18+}\right.$, etc. $)$ are in progress.

\section{ACKNOWLEDGMENTS}

We acknowledge the support from Fundação de Amparo a Pesquisa do Estado de São Paulo of Brazil. The numerical calculations were performed on the IBM SP2 Supercomputer of the Departamento de Física-IBILCE - UNESP, São José do Rio Preto, Brazil.
[1] P. Ackerbauer, J. Werner, W.H. Breunlich, M. Cargnelli, S. Fussy, M. Jeitler, P. Kammel, J. Marton, A. Scrinzi, J. Zmeskal, J. Bistirlich, K.M. Crowe, J. Kurck, C. Petitjean, R.H. Sherman, H. Bossy, H. Daniel, F.J. Hartmann, W. Neumann, G. Schmidt, and M.P. Faifman, Nucl. Phys. A 652, 311 (1999).

[2] R. Jacot-Guillarmod, F. Mulhauser, C. Piller, L.A. Schaller, L. Schellenberg, H. Schneuwly, Y.A. Thalmann, S. Tresch, A. Werthmüller, and A. Adamczak A, Phys. Rev. A 55, 3447 (1997); results for carbon on page 3449.

[3] F. Mulhauser and H. Schneuwly, J. Phys. B 26, 4307 (1993).

[4] A. Werthmüller, A. Adamczak, R. Jacot-Guillarmod, F. Mulhauser, L.A. Schaller, L. Schellenberg, H. Schneuwly, Y.A. Thalmann, and S. Tresch, Hyperfine Interact. 116, 1 (1998).

[5] R. Jacot-Guillarmod, Phys. Rev. A 51, 2179 (1995).

[6] Y.-A. Thalmann, R. Jacot-Guillarmod, F. Mulhauser, L.A. Schaller, L. Schellenberg, H. Schneuwly, S. Tresch, and A. Wertmüller, Phys. Rev. A 57, 1713 (1998).

[7] S.G. Basiladze, P.F. Ermolov, and K.O. Oganesyan, Zh. Eksp. Teor. Fiz. 49, 1042 (1965) [Sov. Phys. JETP 22, 725 (1966)].

[8] P.K. Haff, E. Rodrigo, and T.A. Tombrello, Ann. Phys. (N.Y.) 104, 363 (1977).

[9] L. Schellenberg, Hyperfine Interact. 82, 513 (1993).

[10] A. Bertin, M. Bruno, A. Vitale, A. Placci, and E. Zavattini, Phys. Rev. A 7, 462 (1973).

[11] S.S. Gershtein, Zh. Eksp. Teor. Fiz. 43, 706 (1962) [Sov. Phys. JETP 16, 501 (1963)].

[12] Y. Hahn, Phys. Rev. 169, 794 (1968).

[13] Y. Hahn and K.M. Watson, Phys. Rev. A 5, 1718 (1972).
[14] R.A. Sultanov and S.K. Adhikari, Phys. Rev. A 61, 022711 (2000).

[15] R.A. Sultanov and S.K. Adhikari, J. Phys. B 32, 5751 (1999).

[16] R. A. Sultanov and S. K. Adhikari, Nucl. Phys. A (to be published).

[17] R.A. Sultanov, W. Sandhas, and V.B. Belyaev, Eur. Phys. J. D 5, 33 (1999).

[18] R.A. Sultanov, Few-Body Syst., Suppl. 10, 281 (1999); Innovative Computational Methods in Nuclear Many-Body Problems, edited by H. Horiuchi, Y. Fujiwara, M. Matsuo, M. Kamimua, H. Toki, and Y. Sakuragi (World Scientific, Singapore, 1998), p. 131.

[19] L. Castillejo, I.C. Percival, and M.J. Seaton, Proc. R. Soc. London, Ser. A 254, 259 (1960).

[20] P.G. Burke and K. Smith, Rev. Mod. Phys. 34, 458 (1962), see p. 465.

[21] A.Yu. Voronin and J. Carbonell, Phys. Rev. A 57, 4335 (1998).

[22] W. Tobocman, Theory of Direct Nuclear Reactions (Oxford University Press, London, 1961).

[23] N.F. Mott and H.S.W. Massey, The Theory of Atomic Collisions (Oxford University Press, London, 1965).

[24] R.K. Nesbet, Variational Methods in Electron-Atom Scattering Theory (Plenum Press, New York, 1980); S.K. Adhikari, Variational Principles and the Numerical Solution of Scattering Problems (John Wiley and Sons, New York, 1998).

[25] M. Abramowitz and I.A. Stegun, in Handbook of Mathematical Functions (Dover Publications, New York, 1968), p. 884, Eq. (25.3.23), and p. 885, Eq. (25.4.1). 